\title{
LARGE INTERVALS IN THE CLONE LATTICE
}

\author{
MARTIN GOLDSTERN AND SAHARON SHELAH
}

\begin{abstract}
We give three examples of large intervals in the lattice of (local) clones on an infinite set $X$, by exhibiting clones $\mathscr{C}_{1}, \mathscr{C}_{2}, \mathscr{C}_{3}$ such that:

(1) the interval $\left[\mathscr{C}_{1}, \mathscr{O}\right]$ in the lattice of local clones is (as a lattice) isomorphic to $\{0,1,2, \ldots\}$ under the divisibility relation,

(2) the interval $\left[\mathscr{C}_{2}, \mathscr{O}\right]$ in the lattice of local clones is isomorphic to the congruence lattice of an arbitrary semilattice,

(3) the interval $\left[\mathscr{C}_{3}, \mathscr{O}\right]$ in the lattice of all clones is isomorphic to the lattice of all filters on $X$

These examples explain the difficulty of obtaining a satisfactory analysis of the clone lattice on infinite sets. In particular, (1) shows that the lattice of local clones is not dually atomic.
\end{abstract}

\section{InTRODUCTION}

Definition 0.1. Let $X$ be a nonempty set. The full clone on $X$, called $\mathscr{O}$ or $\mathscr{O}(X)$ is the set of all finitary functions or ("operations") on $X: \mathscr{O}=\bigcup_{n=1}^{\infty} \mathscr{O}^{(n)}$, where $\mathscr{O}^{(n)}$ is the set of all functions from $X^{n}$ into $X$.

A clone (on $X$ ) is a set $\mathscr{C} \subseteq \mathscr{O}$ which contains all projections and is closed under composition.

Alternatively, $\mathscr{C}$ is a clone if $\mathscr{C}$ is the set of term functions of some universal algebra over $X$.

For any set $\mathscr{C} \subseteq \mathscr{O}$, we write $\operatorname{cl}(\mathscr{C})$ for the smallest clone containing $\mathscr{C}$.

The set of clones over $X$ forms a complete algebraic lattice with largest element $\mathscr{O}$. The coatoms of this lattice are called "precomplete clones" or "maximal clones". (See also Szendrei 1986, Pöschel+Kalužnin 1979).

Definition 0.2. A clone $\mathscr{C}$ is called a local clone, iff each set $\mathscr{C} \cap \mathscr{O}^{(k)}$ is closed in the product topology (Tychonoff topology) on $X^{X^{k}}$, where $X$ is taken to be discrete. In other words, $\mathscr{C}$ is local iff:

Whenever $f \in \mathscr{O}^{(k)}$, and for all finite sets $A \subseteq X^{k}$ there is $g \in \mathscr{C}$ with $g \uparrow A=f \uparrow A$, then $f \in \mathscr{C}$

For any $\mathscr{C} \subseteq \mathscr{O}$, we write $\operatorname{loc}(\mathscr{C})$ for the smallest local clone containing $\mathscr{C}$. (So $\operatorname{cl}(\mathscr{C}) \subseteq l o c(\mathscr{C})$.)

Date: 2000-07-30; 2001-01-12; 2001-09-24; 2002-08-08; 2004-07-28.

Key words and phrases. precomplete clones; local clones; dually atomic.

The first author is grateful to the Hebrew University of Jerusalem for the hospitality during his visit, and to the Austrian Science foundation for supporting the joint research under FWF grant P13325-MAT.

The second author is supported by the German-Israeli Foundation for Scientific Research \& Development Grant No. G-294.081.06/93. Publication number 747. 
The set of local clones over $X$ forms again a complete lattice with largest element $\mathscr{O}$ (a sublattice of the lattice of all clone). The coatoms of this lattice are called "precomplete local clones" or "maximal local clones". Note that not every precomplete local clone is also a precomplete clone.

If $X$ is finite (so the notions of "clone" and "local clone" coincide), the precomplete clones are useful as a completeness criterion. By a theorem of Rosenberg,

- there are only finitely many (how many depends on the size of $X$ ) precomplete clones, and in fact there is an explicit list $\mathscr{M}_{1}^{X}, \ldots, \mathscr{M}_{m}^{X}$ of them, together with effective procedures for checking $f \in \mathscr{M}_{i}$

- every clone $\mathscr{C} \neq \mathscr{O}$ is contained in a precomplete clone.

Hence, there is an effective procedure to check if a given set of functions generates all of $\mathscr{O}$, i.e., $\operatorname{cl}\left(\left\{f_{1}, \ldots, f_{n}\right\}\right)=\mathscr{O}$ : Just check if $\left\{f_{1}, \ldots, f_{n}\right\}$ is contained in one of the maximal clones.

For an infinite set $X$, it is known that there are infinitely many precomplete clones (in fact: $2^{|X|}$ many precomplete local clones, and $2^{2^{|X|}}$ many precomplete clones). The question whether every clone $\neq \mathscr{O}$ on an infinite set $X$ is below a precomplete clone ["Is the lattice of clones dually atomic?"] is still not fully resolved (the answer is "no", if $X$ is countable and the continuum hypothesis holds, see Goldstern+Shelah 2005).

We will show here that the corresponding question for local clones has a negative answer in ZFC.

Rosenberg+Szabó 1984 showed that there are unbounded chains in the lattice of local clones (i.e., chains whose only upper bound is the trivial bound $\mathscr{O}$ ).

However, note that for a partial order $(P, \leq)$ the properties

(*) Every element of $P$ is below a maximal element

(**) Every chain of $P$ is bounded

are in general not equivalent, not even if we restrict our attention to those partial orders which are of the form $L \backslash\{\max L\}$, for a complete algebraic lattice $L$.

The property $(* *)$ always implies $(*)$ (by Zorn's lemma), and the property $(*)$ trivially implies $(* *)$ if $P$ has only finitely many maximal elements.

\section{The lattice of LOCAL ClOnes is not DUAlly ATOMiC}

On any infinite set $X$ we will define a local clone $\mathscr{C}_{1}$ such that the interval $\left[\mathscr{C}_{1}, \mathscr{O}\right]$ in the local clone lattice is isomorphic to the natural numbers ordered by the divisibility relation.

Setup 1.1. Fix an infinite set $X$, and let $s: X \rightarrow X$ be a 1-1 onto map without cycles. In other words, $X=Y \times \mathbb{Z}$, and $s(y, n)=(y, n+1)$ for all $y \in Y, n \in \mathbb{Z}$. The orbits of $s$ (or: the sets $\{y\} \times \mathbb{Z}$ are called "components".

Notation 1.2. We will write $\bar{a}$ or $\bar{b}$ to denote tuples $\left(a_{1}, \ldots, a_{n}\right)$ or $\left(b_{1}, \ldots b_{k}\right)$ (the values of $n$ or $k$ will be either irrelevant, or clear from the context).

If $\bar{a}, \bar{b}$ are as above, then $(\bar{a}, \bar{b})$ or $\left(\bar{a}, b_{i}: 1 \leq i \leq k\right)$ denotes the $n+k$-tuple $\left(a_{1}, \ldots, a_{n}, b_{1}, \ldots, b_{k}\right)$. 
For $n>0, s^{n}$ is the $n$-th iterate of $s, s^{-n}$ is the inverse of $s^{n} . s^{0}$ is the identity function. For $n \in Z, a \in X$ we may write $a+n$ instead of $s^{n}(a)$.

More generally, if $\bar{a}=\left(a_{1}, \ldots, a_{k}\right), n \in \mathbb{Z}$, then we write $\bar{a}+n$ for $\left(s^{n}\left(a_{1}\right), \ldots, s^{n}\left(a_{k}\right)\right)$. Similarly, we may write $\bar{a}-n$ for $\bar{a}+(-n)$.

Clearly, $\left(\bar{a}+n_{1}\right)+n_{2}=\bar{a}+\left(n_{1}+n_{2}\right)$, and $\left(\bar{a}+n_{1}\right)-n_{2}=$ $\bar{a}+\left(n_{1}-n_{2}\right)$, so we will often omit parentheses.

Definition and Fact 1.3. For every $n \in \mathbb{N}$, the set

$$
\operatorname{Pol}\left(s^{n}\right):=\bigcup_{k=1}^{\infty}\left\{f \in \mathscr{O}^{(k)}: \forall \bar{a}[f(\bar{a}+n)=f(\bar{a})+n]\right\}
$$

is a local clone.

For $n=0, \operatorname{Pol}\left(s^{0}\right)=\mathscr{O}$.

These clones, and also the unbounded chain $\operatorname{Pol}\left(s^{2^{n}}\right)$ were already considered in Rosenberg+Szabó 1984.

Theorem 1.4. Let $s$ be as above. $\mathscr{C}_{1}:=\operatorname{Pol}(s)$. Then the map $n \mapsto \operatorname{Pol}\left(s^{n}\right)$ is a lattice isomorphism between the following two lattices:

- $(\mathbb{N}, \mid)$, the natural numbers with the divisibility relation, where 1 is the smallest and 0 the greatest element

- $\left(\left[\mathscr{C}_{1}, \mathscr{O}\right], \subseteq\right)$, the set of all local clones extending $\mathscr{C}_{1}$; this set is an interval in the lattice of local clones on $X$.

In particular, there is no precomplete local clone above $\mathscr{C}_{1}$. Also, if $s$ has infinitely many components, then $(X, s) \simeq\left(X, s^{n}\right)$ for all $n \neq 0$, so all clones in $\left[\mathscr{C}_{1}, \mathscr{O}\right)$ are isomorphic (i.e., conjugate to each other via permutations of $X$ ).

Definition 1.5. We say that $\bar{a}$ and $\bar{b}$ are parallel (or $s$-parallel),

$$
\bar{a} \| \bar{b} \quad\left(\bar{a} \|_{s} \bar{b}\right)
$$

iff there is some $n \in \mathbb{Z}, \bar{a}+n=\bar{b}$.

Fact 1.6. (1) For each $k, \|$ is an equivalence relation on $X^{k}$.

(2) If $A \subseteq X^{k}$ meets each $\|$-equivalence class in at most one element [exactly one element], and $g: A \rightarrow X$, then there is a function [there is a unique function] $f \in \operatorname{Pol}(s)^{(k)}$ with $f \uparrow A=g$.

Definition 1.7. For any local clone $\mathscr{D} \supseteq \operatorname{Pol}(s)$ define $G_{\mathscr{D}}$ and $n_{\mathscr{D}}$ as follows:

$$
\begin{aligned}
G_{\mathscr{D}} & :=\left\{n \in \mathbb{Z}: \mathscr{D}^{(1)} \subseteq \operatorname{Pol}\left(s^{n}\right)\right\} \\
n_{\mathscr{D}} & :=\min \left\{n \in G_{\mathscr{D}}, n>0\right\}, \quad n_{\mathscr{D}}:=0 \text { if } G_{\mathscr{D}}=\{0\}
\end{aligned}
$$

Fact 1.8. $G_{\mathscr{D}}$ is a subgroup of $\mathbb{Z}$, hence $G_{\mathscr{D}}=\left\{n_{\mathscr{D}} \cdot k: k \in \mathbb{Z}\right\}$

Proof. Let $n, m \in G_{\mathscr{D}}, f \in \mathscr{D}^{(1)}$. We have to check $f(x+n-m)=$ $f(x)+n-m$ for all $x \in X$ :

$$
f(x+(n-m))=f((x+n)-m)=f(x+n)-m=f(x)+n-m
$$

Lemma 1.9. If $\mathscr{D} \supseteq \operatorname{Pol}(s)$ is a clone, and $\mathscr{D} \neq \operatorname{Pol}(s)$, then already $\mathscr{D}^{(1)} \neq \operatorname{Pol}(s)^{(1)}$. 
Proof. Let $g \in \mathscr{D} \cap \mathscr{O}^{(k)} \backslash \operatorname{Pol}(s)$, say $g(\bar{a}+1) \neq g(\bar{a})+1$. Fix any $b \in X$. We can find unary functions $f_{1}, \ldots, f_{k} \in \operatorname{Pol}(s)$ such that $f_{i}(b)=a_{i}$.

Now consider the function $h: X \rightarrow X$, defined by

$$
h(x)=g\left(f_{1}(x), \ldots, f_{k}(x)\right)
$$

Clearly $h \in \mathscr{D}^{(1)}$, and $h(b+1)=g(\bar{a}+1) \neq g(\bar{a})+1=h(b)+1$, so $h \notin \operatorname{Pol}(s)$.

Lemma 1.10. Let $\mathscr{E} \subseteq \mathscr{O}^{(1)}$, and let $\mathscr{D}$ be the local clone generated by $\operatorname{Pol}(s) \cup \mathscr{E}, n^{*}:=n_{\mathscr{D}}$.

If $\bar{a}=\left(a_{1}, \ldots, a_{k}\right)$ and $\bar{b}=\left(b_{1}, \ldots, b_{k}\right)$ are not $s^{n^{*}}$-parallel, then there is a function $f \in \mathscr{D}$ such that $(\bar{a}, f(\bar{a}))$ and $(\bar{b}, f(\bar{b}))$ are not $s$-parallel.

Proof. First, note that $\operatorname{Pol}(s) \cup \mathscr{D}^{(1)} \subseteq \operatorname{Pol}\left(s^{n^{*}}\right)$, so also $\mathscr{D} \subseteq \operatorname{Pol}\left(s^{n^{*}}\right)$.

If $\bar{a}$ and $\bar{b}$ are not $s$-parallel, then there is nothing to do, so assume $\bar{b}=\bar{a}+\ell$. By our assumption, $\ell$ is not divisible by $n^{*}$, so $\ell \notin G_{\mathscr{D}}$.

We can find a function $d \in \mathscr{D}^{(1)}$ and some $c \in X$ with $d(c+\ell) \neq$ $d(c)+\ell$.

By fact 1.6 there is a function $g \in \operatorname{Pol}(s)$ with $g\left(a_{1}\right)=c\left(\right.$ and $g\left(a_{1}+\right.$ $\ell)=c+\ell$ ).

Now let $f:=d \circ g \circ \pi_{1}^{n}$, then $f(\bar{a})=d(c), f(\bar{a}+\ell)=d(c+\ell) \neq$ $d(c)+\ell=f(\bar{a})+\ell$.

Proof of theorem 1.4. It is clear that the map $n \mapsto \operatorname{Pol}\left(s^{n}\right)$ maps natural numbers to local clones above $\operatorname{Pol}(s)$, and that this map is 1-1. It remains to show that this map is onto.

So let $\mathscr{E} \supseteq \operatorname{Pol}(s)$ be a local clone. We will first consider the clone $\mathscr{D}=\operatorname{loc}\left(\mathscr{E}(\overline{1)} \cap \operatorname{Pol}(s))\right.$ and prove that $\mathscr{D}=\operatorname{Pol}\left(s^{n}\right)$ for some $n \in \mathbb{N}$. If $n=0$, then $\mathscr{D}=\mathscr{E}=\mathscr{O}$, and if $n>0$ then we invoke lemma 1.9] to show $\mathscr{E}=\mathscr{D}$.

So we are now looking at a clone $\mathscr{D} \supsetneq \operatorname{Pol}(s)$, where $\mathscr{D}=\operatorname{loc}\left(\mathscr{D}^{(1)} \cup\right.$ $\operatorname{Pol}(s))$.

Define $G_{\mathscr{D}}$ and $n^{*}:=n_{\mathscr{D}}$ as in definition 1.7

We will prove $\operatorname{Pol}\left(s^{n^{*}}\right) \subseteq \mathscr{D}$. Since $\mathscr{D}$ is a local clone, it is enough to show that every function in $\operatorname{Pol}\left(s^{n^{*}}\right)$ can be interpolated by a function in $\mathscr{D}$ on any finite set.

So let $g \in \operatorname{Pol}\left(s^{n^{*}}\right)$ be $k$-ary.

Let $\bar{a}_{1}, \ldots, \bar{a}_{n}$ be in $X^{k}, b_{\ell}:=g\left(\bar{a}_{\ell}\right)$. We claim that there is a function $f \in \mathscr{D}$ satisfying also $b_{\ell}:=f\left(\bar{a}_{\ell}\right)$ for $\ell=1, \ldots, n$.

Wlog we may assume that no two of the $k$-tuples $\bar{a}_{\ell}$ are $s^{n^{*}}$ parallel. [This assumption is allowed, since $g \in \operatorname{Pol}\left(s^{n^{*}}\right)$.]

Let $I$ be the set of all pairs $i=\left(\ell_{1}, \ell_{2}\right)$ of distinct numbers in $\{1, \ldots, n\}$. For each $i=\left(\ell_{1}, \ell_{2}\right) \in I$ we can find a function $f_{i} \in \mathscr{D}$ such that $\left(\bar{a}_{\ell_{1}}, f_{i}\left(\bar{a}_{\ell_{1}}\right)\right)$ and $\left(\bar{a}_{\ell_{2}}, f_{i}\left(\bar{a}_{\ell_{2}}\right)\right)$ are not $s$-parallel.

Let $\bar{c}_{\ell}:=\left(\bar{a}_{\ell}, f_{i}\left(\bar{a}_{\ell}\right): i \in I\right)$. Clearly, for all $\ell_{1} \neq \ell_{2}$ we have: $\bar{c}_{\ell_{1}}$ and $\bar{c}_{\ell_{2}}$ are not $s$-parallel.

So by fact 1.6 there is a function $h \in \operatorname{Pol}(s)$ such that

$$
\forall \ell: \quad h\left(\bar{c}_{\ell}\right)=b_{\ell}
$$

So the function $f$ defined by

$$
f(\bar{x})=h\left(\bar{x}, f_{i}(\bar{x}): i \in I\right)
$$

is in $\mathscr{D}$, and it satisfies $f\left(\bar{a}_{\ell}\right)=b_{\ell}$ for all $\ell$. 
Remark 1.11. Let $H$ be any constant function. Then $\operatorname{cl}(\operatorname{Pol}(s) \cup$ $\{H\})=\mathscr{O}$. It is well known (and easy to show, using Zorn's lemma) that this implies that every clone above $\operatorname{Pol}(s)$ is below some precomplete clone.

(In particular, also the (nonlocal) clone $\mathscr{C}_{\infty}:=\bigcup_{n>0} \operatorname{Pol}\left(s^{n}\right)$ is below a precomplete clone.)

\section{A large interval of LOCAL ClONES}

Theorem 2.1. Let $\mathfrak{S}=(S, \vee)$ be a downward directed semilattice, and let $\operatorname{Con}(\mathfrak{S})$ be the lattice of congruences on $\mathfrak{S}$. Then there is a local clone $\mathscr{C}_{2}$ (on the set $S$ ) such that

$$
\left[\mathscr{C}_{2}, \mathscr{O}_{S}\right] \simeq \operatorname{Con}(\mathfrak{S})
$$

That is, there is a lattice isomorphism between the set of local clones above $\mathscr{C}$ and the set of congruences of $\mathfrak{S}$.

Remark: If $\emptyset \subsetneq I \subsetneq S$ is an ideal, then the partition $\{I, S \backslash I\}$ corresponds to a congruence relation which is a coatom in $\operatorname{Con}(\mathfrak{S})$. In fact, all coatoms are obtained in this form. It is clear that $\operatorname{Con}(\mathfrak{S})$ is dually atomic.

Definition 2.2. Let $S$ be a set $A \subseteq S$. We let $\operatorname{Pol}(A)$ be the set of all functions on $S$ which "preserve" $A$ :

$$
\operatorname{Pol}(A):=\bigcup_{n=1}^{\infty}\left\{f \in \mathscr{O}^{(n)}: f\left[A^{n}\right] \subseteq A\right\}
$$

Fact 2.3. $\operatorname{Pol}(A)$ is always a local clone.

If $\emptyset \neq A \neq S$, then $\operatorname{Pol}(A)$ is a maximal clone.

Definition and Fact 2.4. Let $\mathfrak{S}=(S, \vee)$ be a semilattice. We call $R \subseteq S \times S$ a congruence order on $\mathfrak{S}$ iff one (or both) of the following two equivalent conditions are satisfied:

(1) $\theta_{R}:=\{(x, y): x R y$ and $y R x\}$ is a (semilattice) congruence relation, and: $x R y$ iff $x / \theta_{R} \leq y / \theta_{R}$.

(2) $R$ is reflexive and transitive, $x \leq y \Rightarrow x R y$, and

$$
\forall x, y, z: \quad x R z \& y R z \Rightarrow(x \vee y) R z
$$

The following fact is trivial:

Fact 2.5. The maps $R \mapsto \theta_{R}$ and $\theta \mapsto\{(x, y): x / \theta \leq y / \theta\}$ are monotone bijections between congruence relations and congruence orders, and they are inverses of each other.

Notation 2.6. For $a, b \in S$ let $\chi_{a, b}$ be the function satisfying $\chi_{a, b}(b)=$ $a, \chi_{a, b}(x)=x$ for $x \neq b$.

Notation 2.7. For $\bar{a}=\left(a_{1}, \ldots, a_{k}\right)$, write $\bigvee \bar{a}$ for $a_{1} \vee \cdots \vee a_{k}$.

Definition and Fact 2.8. Let $\mathfrak{S}=(S, \vee)$ be a semilattice, and let $\sqsubseteq$ be a congruence order on $\mathfrak{S}$. Then

$$
\mathscr{E}(\sqsubseteq):=\bigcup_{k=1}^{\infty}\left\{f \in \mathscr{O}^{(k)}: \forall \bar{x} \in S^{k}[f(\bar{x}) \sqsubseteq \bigvee \bar{x}]\right\}
$$

is a local clone. Furthermore,

(1) $\mathscr{E}(\sqsubseteq)=\bigcap_{a \in S} \operatorname{Pol}\{x \in S: x \sqsubseteq a\}$

(2) $\sqsubseteq_{1} \subseteq \sqsubseteq_{2}$ implies $\mathscr{E}\left(\sqsubseteq_{1}\right) \subseteq \mathscr{E}\left(\sqsubseteq_{2}\right)$.

(3) $f \in \mathscr{E}(\sqsubseteq) \cap \mathscr{O}^{(1)}$ implies $\forall x: f(x) \sqsubseteq x$. 
(4) $\chi_{a, b} \in \mathscr{E}(\sqsubseteq)$ iff $a \sqsubseteq b$.

We will now consider local clones above the clone $\mathscr{C}_{2}:=\mathscr{E}(\leq$ ) and we will show that they all are induced by congruence orders/congruence relations, and that also conversely every congruence relation is induced by a clone.

Definition and Fact 2.9. Let $\mathscr{C} \supseteq \mathscr{C}_{2}$ be a local clone. Then

$$
\sqsubseteq_{\mathscr{C}}:=\left\{(x, y): \exists f \in \mathscr{C}^{(1)} f(y)=x\right\}
$$

is a congruence order.

Also, $\mathscr{C}_{2} \subseteq \mathscr{C}_{1} \subseteq \mathscr{C}_{2}$ implies $\sqsubseteq \mathscr{C}_{1} \subseteq \check{\mathscr{C}}_{2}$.

Proof. We will check condition (2) from definition 2.4 Clearly $\sqsubseteq$ is reflexive and transitive. If $a \leq b$, then the function $\chi_{a, b} \in \mathscr{C}_{2} \subseteq \mathscr{C}$ will witness that $a \sqsubseteq_{\mathscr{C}} b$.

It remains to check $(*)$. So let $a \sqsubseteq c, b \sqsubseteq c$. There are functions $f, g \in \mathscr{C}$ with $f(c)=a, g(c)=b$. Since the function $\vee:(x, y) \mapsto$ $x \vee y$ is in $\mathscr{C}_{2} \subseteq \mathscr{C}$, we also have $(f \vee g) \in \mathscr{C}$, and $f \vee g$ witnesses $a \vee b \Xi_{C} c$.

Lemma 2.10. Let $R$ be a congruence order. Then $R=\sqsubseteq_{\mathscr{E}(R)}$.

Proof. The inclusion $\sqsubseteq_{\mathscr{E}(R)} \subseteq R$ is trivial: Let $a \sqsubseteq_{\mathscr{E}(R)} b$. So there is $f \in \mathscr{E}(R), f(b)=a$. Now by fact 2.8 (3), bRa.

For the proof of the reverse inclusion, $R \subseteq \sqsubseteq_{\mathscr{E}(R)}$, consider any $a R b$. The function $\chi_{a, b} \in \mathscr{E}(R)$ witnesses $a \sqsubseteq_{\mathscr{E}(R)} b$.

Lemma 2.11. Let $\mathscr{C} \supseteq \mathscr{C}_{2}$ be a local clone. Then $\mathscr{C}=\mathscr{E}\left(\varpi_{\mathscr{C}}\right)$.

Proof. The inclusion $\mathscr{C} \subseteq \mathscr{E}\left(\Xi_{\mathscr{C}}\right)$ is trivial: For $f \in \mathscr{C}$ we need to show that for all $a \in S, f$ preserves the set $\{x: x \sqsubseteq \mathscr{C} a\}$. Let $\bar{x}=$ $\left(x_{1}, \ldots, x_{k}\right)$. If $x_{1}, \ldots, x_{k} \sqsubseteq \mathscr{C} a$ then for each $i$ there is some $g_{i} \in \mathscr{C}$ with $g_{i}(a)=x_{i}$. Now $f\left(g_{1}, \ldots, g_{k}\right)$ witnesses that also $f(\bar{x}) \sqsubseteq_{\mathscr{C}} a$.

Now we will show $\mathscr{E}\left(\Xi_{\mathscr{C}}\right) \subseteq \mathscr{C}$ :

Let $f \in \mathscr{E}\left(\complement_{\mathscr{C}}\right)$ be $k$-ary, where $\mathscr{C} \supseteq \mathscr{C}_{2}$. To show that $f \in \mathscr{C}$ it is enough [since $\mathscr{C}$ is local] to show that $f$ can be interpolated by an element of $\mathscr{C}$ on any finite number of places .

So let $\bar{a}_{1}, \ldots, \bar{a}_{n} \in S^{k}$, and let $b_{i}:=f\left(\bar{a}_{i}\right)$. Let $d_{i}:=\bigvee \bar{a}_{i}$.

Since $f \in \mathscr{E}\left(\sqsubseteq_{\mathscr{C}}\right)$, we have $b_{i} \sqsubseteq_{\mathscr{C}} d_{i}$, so there is (for every $i$ ) a unary function $g_{i} \in \mathscr{C}$ with $g_{i}\left(d_{i}\right)=b_{i}$.

Define a $k+1$-ary function $h_{i}$ by letting $h_{i}(y, \bar{x})=y$ if $\bar{x}=\bar{a}_{i}$, and

if $\bar{x} \neq \bar{a}_{i}$, then:

$h(y, \bar{x}):=$ some value which is $\leq x_{j}$ and $\leq b_{j}$ for all $j$, and also $\leq y$.

(It is possible to find such a value, since $\mathfrak{S}$ is downward directed.) Clearly $h_{i} \in \mathscr{C} \subseteq \mathscr{C}$. So the function

$$
f_{i}: \bar{x} \mapsto h_{i}\left(g_{i}(\bigvee \bar{x}), \bar{x}\right)
$$

is in $\mathscr{C}$. Now check that the function $f^{\prime}:=\bigvee_{i=1}^{n} f_{i}$ maps $\bar{a}_{i}$ to $b_{i}$. Clearly $f^{\prime} \in \mathscr{C}$.

Example 2.12. Let $(S,<)$ be a linearly ordered set. Then the congruence relations on $(S, \max )$ are exactly the equivalnce relations with convex classes. 
Example 2.13. As a special case, consider the semilattice ( $\mathbb{N}, \max$ ). A congruence relation is just a partition of $\mathbb{N}$ into disjoint intervals.

The map

$$
\theta \mapsto A_{\theta}:=\{\max E: E \text { is a finite congruence class }\}
$$

is an antitone 1-1 map from the congruence relations into $\mathscr{P}(\mathbb{N})$, the power set of $\mathbb{N}$.

It is also easy to see that this map is onto: Each $A \subseteq \mathbb{N}$ is equal to $A_{\theta(A)}$, where for $k<n$ we have:

$(k, n) \in \theta(A)$ iff there is no $a \in A, k \leq a<n$

The map $A \mapsto \bigcap_{a \in A} \operatorname{Pol}\{0, \ldots, a\}$ is an isomorphism between $(\mathscr{P}(\mathbb{N}), \supseteq)$ and $\left[\mathscr{C}_{2}, \mathscr{O}\right]$. The empty set corresponds to $\mathscr{O}$, or to the equivalence relation with a single class; the set $\mathbb{N}$ itself corresponds to $\mathscr{C}_{2}$, or to the equivalence with singleton classes.

\section{A large interval of ClONES}

On any infinite set $X$ we will define a clone $\mathscr{C}_{3}$ such that the interval $\left[\mathscr{C}_{3}, \mathscr{O}\right]$ in the full clone lattice is very large (with $2^{2^{|X|}}$ precomplete elements), but still reasonably well understood.

Definition 3.1. For any function $f \in \mathscr{O}^{(n)}$, let $f^{(1)} \in \mathscr{O}^{(1)}$ be defined by $f^{(1)}(x)=f(x, \ldots, x)$.

Definition 3.2. For any function $f \in \mathscr{O}^{(n)}$, we let

$$
\operatorname{fix}(f)=\left\{x: f^{(1)}(x)=x\right\} \quad \operatorname{nix}(f)=\left\{x: f^{(1)}(x) \neq x\right\}
$$

Definition 3.3. Let $\mathscr{F} \subseteq \mathscr{P}(X)$ be a family of sets. We define

$$
\mathscr{C}_{\mathscr{F}}:=\{f \in \mathscr{O}: \operatorname{fix}(f) \in \mathscr{F}\}
$$

Fact 3.4. (1) If $\mathscr{F}$ is a filter, then $\mathscr{C}_{\mathscr{F}}$ is a clone.

(2) If $\mathscr{F} \subseteq \mathscr{F}^{\prime}$, then $\mathscr{C}_{\mathscr{F}} \subseteq \mathscr{C}_{\mathscr{F}}$.

Definition 3.5. Let $\mathscr{C}_{3}:=\mathscr{C}_{\{X\}}$ be the clone of "idempotent" functions, i.e., of all functions satisfying $f(x, \ldots, x)=x$ for all $x$.

Theorem 3.6. The map $\mathscr{F} \rightarrow \mathscr{C}_{\mathscr{F}}$ is an order isomorphism between the set of all filters (including the improper filter $\mathscr{P}(X)$ ) and the set of all clones above $\mathscr{C}_{3}$.

In particular, the precomplete clones above $\mathscr{C}_{3}$ are exactly the clones of the form $\mathscr{C}_{U}$, where $U$ is an ultrafilter on $X$.

We will prove this theorem in several steps, concluding with lemma 3.9 below.

Lemma 3.7. Let $\mathscr{F}$ be a filter, $\mathscr{D} \supsetneq \mathscr{C}_{\mathscr{F}}$. Then $\mathscr{D}^{(1)} \supsetneq \mathscr{C}_{\mathscr{F}}^{(1)}$.

Proof. Let $f \in \mathscr{D} \backslash \mathscr{C}_{\mathscr{F}}$. Then $\operatorname{fix}(f)=\operatorname{fix}\left(f^{(1)}\right) \notin \mathscr{F}$, so $f^{(1)} \in$ $\mathscr{D}^{(1)} \backslash \mathscr{C}_{\mathscr{F}}$.

Lemma 3.8. Assume $f \in \mathscr{D} \supseteq \mathscr{C}_{3}$, and $\operatorname{fix}(f) \subseteq \operatorname{fix}(g), f, g \in \mathscr{O}^{(1)}$. Then $g \in \mathscr{D}$.

Proof. Let

$$
H(x, y)= \begin{cases}g(x) & \text { if } x \neq y \\ x & \text { if } x=y\end{cases}
$$

Clearly $H \in \mathscr{C}_{3}$. For $x \in \operatorname{fix}(f) \subseteq \operatorname{fix}(g)$ we have $H(x, f(x))=$ $H(x, x)=x=g(x)$, and for $x \in \operatorname{nix}(f)$ we have $f(x) \neq x$, so $H(x, f(x))=g(x)$.

So in either case, $H(x, f(x))=g(x)$. 
Lemma 3.9. Let $\mathscr{D}$ be a clone with $\mathscr{C}_{3} \subseteq \mathscr{D}, \mathscr{D} \neq \mathscr{O}$. Then there is a (proper) filter $\mathscr{F}$ such that $\mathscr{D}=\mathscr{C}_{\mathscr{F}}$.

Proof. Let $I:=\{\operatorname{nix}(f): f \in \mathscr{D}\}=\left\{\operatorname{nix}(f): f \in \mathscr{D}^{(1)}\right\}$. We first check that $I$ is an ideal.

If $A=\operatorname{nix}(f), f \in \mathscr{D}$, and $B \subseteq A$, then by lemma 3.8 there is a function $g \in \mathscr{D}$ with $B=\operatorname{nix}(g)$. So $I$ is downward closed.

Now let $A_{\ell}=\operatorname{nix}\left(f_{\ell}\right), f_{\ell} \in \mathscr{D}$ for $\ell=1,2$, and assume that $A_{1} \cap A_{2}=\emptyset$.

Let $B=X \backslash\left(A_{1} \cup A_{2}\right)$. We may assume that either $\left|A_{1}\right| \geq 2$, or $B \neq \emptyset$ (or both).

In either case there is a unary function $f_{1}^{\prime}$ with $\operatorname{nix}\left(f_{1}^{\prime}\right)=A_{1}$, and $f_{1}^{\prime}$ maps $A_{1}$ into $A_{1} \cup B$. By lemma 3.8 $f_{1}^{\prime} \in \mathscr{D}$. So $f_{2} \circ f_{1}^{\prime} \in \mathscr{D}$. Also, $\operatorname{nix}\left(f_{2} \circ f_{1}^{\prime}\right)=A_{1} \cup A_{2}$; this shows that $I$ is an ideal.

Let $\mathscr{F}$ be the filter dual to $I$. Clearly,

$$
f \in \mathscr{D} \Rightarrow \operatorname{fix} f \in \mathscr{F} \Rightarrow f \in \mathscr{C}_{\mathscr{F}} .
$$

For the converse, we first check $\mathscr{C}_{\mathscr{F}} \cap \mathscr{O}^{(1)} \subseteq \mathscr{D}$ :

Let $f \in \mathscr{C}_{\mathscr{F}}$ be unary. So $\operatorname{fix}(f) \in \mathscr{F}$, i.e., there is a function $g \in \mathscr{D}$ with fix $f=$ fix $g$. By lemma $3.8 f \in \mathscr{D}$.

Now take an arbitrary $n$-ary function $f \in \mathscr{C}_{\mathscr{F}}$. We need to show that $f \in \mathscr{D}$. Let $A=\operatorname{nix} f \in I$. Define an $n+1$-ary function $H$ as follows:

$$
H\left(x_{1}, \ldots, x_{n}, y\right)= \begin{cases}x_{1} & \text { if } x_{1}=\cdots=x_{n}=y \\ f\left(x_{1}, \ldots x_{n}\right) & \text { otherwise }\end{cases}
$$

Clearly $H \in \mathscr{C}_{3}$. Note that $f^{(1)} \in \mathscr{D}$, so also the function $\bar{x} \mapsto$ $H\left(\bar{x}, f^{(1)}\left(x_{1}\right)\right)$ is in $\mathscr{D}$.

We now check that $H\left(\bar{x}, f^{(1)}\left(x_{1}\right)\right)=f(\bar{x})$ for all $\bar{x}$. We distinguish three cases:

Case 1: $x_{1}=\cdots=x_{n} \in A=\operatorname{nix}(f)$. So $f^{(1)}\left(x_{1}\right) \neq x_{1}$, hence (by definition of $H$ ) we have $H\left(\bar{x}, f^{(1)}\left(x_{1}\right)\right)=f(\bar{x})$.

Case 2: $x_{1}=\cdots=x_{n} \in \operatorname{fix}(f)$. So $f(\bar{x})=f^{(1)}\left(x_{1}\right)=x_{1}$, and also $H\left(\bar{x}, f^{(1)}\left(x_{1}\right)\right)=x_{1}$.

Case 3: Not all $x_{i}$ are equal. Again, by definition of $H$, we have $H\left(x_{1}, \ldots, x_{n}, f^{(1)}\left(x_{1}\right)\right)=f\left(x_{1}, \ldots, x_{n}\right)$.

This shows that $f \in \mathscr{D}$.

Remark 3.10. If we regard the set $X$ as a discrete topological space, then the Stone-Cech compactification of $X$ is

$$
\beta X=\{U: U \text { is an ultrafilter on } X\}
$$

There is a canonical 1-1 order-preserving correspondence between the filters on $X$ (ordered by $\subseteq$ ) and the closed subsets of $\beta X$ (ordered by $\supseteq$ ).

So the interval $\left[\mathscr{C}_{3}, \mathscr{O}\right]$ in the full clone lattice is isomorphic (as a complete lattice) to the family of closed subsets of $\beta X$, ordered by reverse inclusion: $\mathscr{O}$ corresponds to the empty set, each precomplete clone in $\left[\mathscr{C}_{3}, \mathscr{O}\right]$ corresponds to a singleton set.

Note that for any closed subset $F \subseteq \beta X$ and any $p \in \beta X \backslash F$, also $F \cup\{p\}$ is closed, and moreover:

$F$ covers $G$ (i.e., $F \supset G$, and the interval $(G, F)$ is empty) iff $G=F \cup\{p\}$ for some $p \in \beta X \backslash F$ 
In particular, let $\mathscr{C}_{\text {bd }} \supseteq \mathscr{C}_{3}$ be the clone corresponding to the ideal of small sets, i.e.,

$$
\mathscr{C}_{\mathrm{bd}}:=\left\{f \in \mathscr{O}^{(:)} \exists B \subseteq X,|B|<|X|, \forall x \in X \backslash B: f(x, \ldots, x)=x\right\}
$$

Then every clone $\mathscr{C} \supsetneq \mathscr{C}_{\text {bd }}$ has exactly $2^{2^{|X|}}$ lower neighbors in the clone lattice; the clone corresponds to a closed set $F$, and the lower neighbors correspond to closed sets $F \cup\{p\}$.

This is a special case of a theorem of [Marchenkov 1981].

\section{REFERENCES}

[Freese+Nation 1973] Ralph Freese and J. B. Nation. Congruence lattices of semilattices. Pacific J. Math., 49:51-58, 1973.

[Goldstern+Shelah 2005] Martin Goldstern and Saharon Shelah. Clones from Creatures. Transactions of the American Mathematical Society, accepted. math.RA/0212379

[Marchenkov 1981] S. S. Marchenkov. Cardinality of the set of precomplete classes in certain classes of functions of countably valued logic. Problemy Kibernet., 38:109-116, 272, 1981.

[Pöschel+Kalužnin 1979] R. Pöschel and L. A. Kalužnin. Funktionen- und Relationenalgebren, volume 15 of Mathematische Monographien [Mathematical Monographs]. VEB Deutscher Verlag der Wissenschaften, Berlin, 1979. Ein Kapitel der diskreten Mathematik. [A chapter in discrete mathematics].

[Rosenberg+Szabó 1984] I. G. Rosenberg and L. Szabó. Local completeness. I. Algebra Universalis, 18(3):308-326, 1984.

[Szendrei 1986] Ágnes Szendrei. Clones in universal algebra. Presses de l'Université de Montréal, Montreal, Que., 1986.

[Žitomirskiı̌ 1971] G. I. Žitomirskiǔ. The lattice of all congruence relations on a semilattice. In Ordered sets and lattices, No. 1 (Russian), pages 11-21. Izdat. Saratov. Univ., Saratov, 1971.

DMG/Algebra, TU Wien

WiedNER HauptSTRASSE 8-10/104.1

A-1040 WiEN

E-mail address: Martin.Goldstern@tuwien.ac.at

$U R L$ : http://info.tuwien.ac.at/goldstern/

Mathematics

Hebrew University of Jerusalem

91904 JeRUSALEM, ISRAEL

E-mail address: shelah@math.huji.ac.il

$U R L:$ http://math.rutgers.edu/ shelah 